\title{
Infrastructure Wi-Fi for connected autonomous vehicle positioning: A review of the state-of-the-art
}

\author{
E.I. Adegoke ${ }^{\mathrm{a}, *}$, Jasmine Zidane ${ }^{\mathrm{a}}$, Erik Kampert ${ }^{\mathrm{a}}$, Col R. Ford ${ }^{\mathrm{b}}$, Stewart A. Birrell ${ }^{\mathrm{a}}$, \\ Matthew D. Higgins ${ }^{a}$ \\ a WMG, University of Warwick, Coventry, CV4 7AL, UK \\ b Spirent Communications Plc, TQ4 7QR, Paignton, UK
}

\section{A R T I C L E I N F O}

\section{Article history:}

Received 7 May 2019

Received in revised form 29 August 2019

Accepted 9 September 2019

Available online 17 September 2019

\section{Keywords:}

Positioning

CAVs

Wi-Fi

GNSS

Intelligent Transportation Systems

Vehicle-to-vehicle/roadside/Internet

communication

\begin{abstract}
A B S T R A C T
In order to realize intelligent vehicular transport networks and self driving cars, connected autonomous vehicles (CAVs) are required to be able to estimate their position to the nearest centimeter. Traditional positioning in CAVs is realized by using a global navigation satellite system (GNSS) such as global positioning system (GPS) or by fusing weighted location parameters from a GNSS with an inertial navigation systems (INSs). In urban environments where Wi-Fi coverage is ubiquitous and GNSS signals experience signal blockage, multipath or non line-of-sight (NLOS) propagation, enterprise or carrier-grade Wi-Fi networks can be opportunistically used for localization or "fused" with GNSS to improve the localization accuracy and precision. While GNSS-free localization systems are in the literature, a survey of vehicle localization from the perspective of a Wi-Fi anchor/infrastructure is limited. Consequently, this review seeks to investigate recent technological advances relating to positioning techniques between an ego vehicle and a vehicular network infrastructure. Also discussed in this paper is an analysis of the location accuracy, complexity and applicability of surveyed literature with respect to intelligent transportation system requirements for CAVs. It is envisaged that hybrid vehicular localization systems will enable pervasive localization services for CAVs as they travel through urban canyons, dense foliage or multi-story car parks.
\end{abstract}

(C) 2019 The Author(s). Published by Elsevier Inc. This is an open access article under the CC BY-NC-ND license (http://creativecommons.org/licenses/by-nc-nd/4.0/).

\section{Introduction}

By integrating information communication technologies into transport networks, the next generation of transportation systems will become safer, more efficient and sustainable. A European initiative known as intelligent transport systems (ITS) aims to create a manageable transport network that will foster increased penetration of automation within the industry. Ongoing research in ITS span from dynamic traffic management in road networks to telematics applications for freight services in rail traffic systems [1]. In order to increase automation in road transport networks and improve passenger safety, vehicles and road traffic management systems need to be interconnected and internetworked. At the highest level of vehicular automation, connected autonomous vehicles

\footnotetext{
th This work has been sponsored by Innovate UK under grant No. 95143-564624 with Spirent Communications Plc., WMG and Chronos Technology Ltd. The doctorate of J. Zidan is also supported by Spirent Communications Plc. as part of the Warwick Collaborative Postgraduate Research Scholarships (WCPRS)

* Corresponding author.

E-mail address: elijah.adegoke@warwick.ac.uk (E.I. Adegoke).
}

(CAVs) will usher in self-driving cars interconnected to transportation infrastructure and vehicular networks using wireless communications. In order for CAVs to be truly autonomous for navigation, it is essential that the vehicle is aware of its position, surrounding environment and nearby vehicles [2]. Irrespective of the architecture, CAVs are essentially made up of five subsystems, which are: localization, perception, planning, control and system management [3]. With respect to localization (which is also referred to as positioning), satellite based systems such as GNSS have been widely adopted by automobile original equipment manufacturers (OEMs) for absolute vehicle positioning. However, some other navigation methods such as inertial measurement units (IMUs), cameras [4,5], Radar [6], wireless wide area networks (WWANs) such as $3 \mathrm{G} / 4 \mathrm{G} / 5 \mathrm{G}$ [7-9] or Wi-Fi have also been adopted in CAVs for relative positioning

IMUs are able to provide information relating to the relative position of a vehicle by measuring linear and angular motion using odometers, gyroscopes and accelerometers. By combining the trajectory information obtained from the IMU with the reference initial position of the vehicle (obtained from a GNSS), kinematic equations can be used to estimate the current position of the 
ego/tracked vehicle. The drawbacks of this technique are the additional hardware cost incurred and the accumulated positioning error [2]. This position estimation technique has been adopted in vehicle and human localization $[10,11]$.

Perception based techniques can also be used to estimate the position of a CAV. By solely using cameras on vehicles, images or video content can be captured or recorded and used for localization. This technique can be carried out using digital maps generated a priori or by using simultaneous localization and mapping (SLAM). In the former, a high definition digital map (in the form of local dynamic map [12] or point-cloud) is fed alongside the captured data into a statistical estimation algorithm (such as Bayes filter) to estimate the vehicle's location [13]. The latter constructs a map of a given space while also using the same map for localization/navigation. In point-cloud vision based methods, data capture is via cameras or light detection and ranging (LiDAR) and a GNSS based system is adopted in planar maps. The major challenges associated with this navigation method are weather impairments and the need for high computational processing.

Due to multipath and interference associated with GNSS signal reception in urban environments/canyons and strict CAV requirements (such as ms end-to-end latency, cm accuracy and 99.999\% availability) [14], it is evident that real-time localization of a vehicle using a singular technique becomes a challenging task. A pragmatic approach adopted in the literature is to "fuse" location data from WWANs, perception sensors, IMUs or wireless sensor networks (WSNs) with GNSS signals in order to accurately determine a vehicle's position. In most urban areas where wireless network coverage is good, wireless networks can be used opportunistically for positioning/localization. Vehicle positioning can also be obtained from the opportunistic use of IEEE 802.11p vehicleto-everything (V2X) communications [15], which has been implemented as ETSI ITS G5 [16] and IEEE 1609 dedicated short-range communications (DSRC) [17]. Alternatively, the 3GPP is also developing cellular V2X communications (C-V2X). Typical V2X deployment scenarios will involve the installation of transceivers in the vehicle as onboard units (OBU) and in road side units (RSU) within the environment. Given that V2X technology is primarily designed for communications, opportunistic usage can be realized when RSUs share clock and location information. Vehicle Localization via this approach will require both the OBU and RSU clocks to be synchronized. This will introduce additional cost as the timing hardware in both units will require significant upgrades. While similar wireless ranging techniques can be adopted, Wi-Fi access points (APs) and mobile network base station transceivers (BSs) have different characteristics with respect to anchor density (number of anchors per unit area), coverage, antenna system and RF front end. As a result, the accuracy obtainable from these wireless networks will differ. For example, in a time/delay based ranging technique; the physical (PHY) layer implementation in Wi-Fi and $4 \mathrm{G} / 5 \mathrm{G}$ networks differ with respect to preamble lengths.

Since that the performance of GNSS is impaired with the absence of line-of-sight (LOS) signals as evident in urban canyons or within car parks, optimally combining complementary position data sources becomes paramount. In the literature (as shown in Section 3), Bayesian filtering techniques are usually adopted in estimating the state space of the ego vehicle. For non-stationary systems as a vehicle, these techniques are based on modeling the noise and vehicle dynamics by using linear/non-linear or Gaussian/non-Gaussian theories. Some of the filtering techniques used in the literature surveyed include: Kalman Filter (KF), Extended Kalman Filter (EKF), Particle Filter (PF) and Unscented Kalman Filter (UKF).

Existing surveys (such as $[18,19]$ ) address a plethora of positioning methods for CAVs. This review seeks to focus on the stateof-the-art of infrastructure based Wi-Fi CAV localization as well as hybrid GNSS localization systems that are coupled with Wi-Fi infrastructure. Moreover further contribution is presented through a descriptive comparison of the surveyed works with respect to ease of implementation, cost, as well as performance of the localization frameworks. In addition, survey conclusions are presented and future research areas are discussed and recommended.

The remainder of this paper is organized as follows: Section 2 discusses widely known wireless ranging methods and performance criteria used for evaluating these systems. Section 3 presents recent Wi-Fi based CAV localization techniques as well as their accompanying position estimation algorithms. In Section 4 we conclude the review and recommend areas for research.

\section{Wireless ranging, sensor fusion and GNSS performance criteria}

\subsection{Wireless ranging}

Wireless/radio ranging is premised on the idea that the distance between a transmitter and a receiving device can be inferred from the properties of the radio wave observed at the receiver. Wireless ranging can be implemented using three widely known methods: receive signal strength (RSS), ${ }^{1}$ arrival time and arrival angle. In order to improve the ranging accuracy at the expense of additional complexity, some localization frameworks adopt two or more wireless ranging techniques [21].

\subsubsection{RSS-based}

This ranging method estimates the separation distance from the received signal power measurements. From a wireless propagation perspective, radio waves will experience reflection, diffraction and scattering as they travel through the wireless channel. These effects cause the received signal to gradually vary over an arbitrary transmitter-receiver (T-R) separation distance and/or rapidly vary over a few wavelengths. The propagation effects relating to the former are known as large-scale fading and small-scale/multipath fading for the latter $[22,23]$. With respect to wireless positioning systems (WPS), RSS radio ranging can be implemented as access point/base station (AP/BS) trilateration using path loss models or Wi-Fi fingerprinting as illustrated in Fig. 1 [24,25]. In terrestrial radio communications, propagation models are typically adopted in predicting the received power or radio coverage. As a result, localization errors usually arise when an inaccurate power-distance model is used to estimate the T-R distance. The most widely used propagation models in WPS are: free space path loss, two-way ground reflection and log-distance shadowing as shown in (1) to (5), where $d$ is the T-R separation distance, $\lambda$ is the wavelength, $h_{t}$ and $h_{r}$ are the transmitter and receiver heights. Given that the two-way ground model gives unstable results for short T-R distances, a crossover distance $\left(d_{c}\right)$ is defined in (4). The first-order log distance path loss model in (5) includes a Gaussian random variable with zero mean and variance $\left(\sigma^{2}\right)$ which accounts for shadowing and clutter in the wireless channel. It also includes path loss at a reference distance $\left(d_{0}\right)$ of $1 \mathrm{~m}$ which can be obtained from measurement data. Thus, measurement-based path loss models (as found in $[26,27]$ ) can be adopted to improve the localization accuracy of AP/BS RSS radio ranging.

$L_{\mathrm{FSPL}}[d B]=20 \log _{10}\left(\frac{4 \pi d}{\lambda}\right)$

\footnotetext{
1 Receive signal strength indicator (RSSI) can also be used as a metric for the received signal strength. However, the estimation of this value is vendor specific and curve fitting using spectrum analyzer power measurements can be used to transform these values [20]. These terms are used interchangeably in this paper without loss of generality.
} 
$L_{2 \mathrm{GR}}[d B]=20 \log _{10}\left(\frac{d^{2}}{h_{t} h_{r}}\right)$

$L_{\text {pathloss }}[d B]= \begin{cases}L_{\mathrm{FSPL}}[d B], & \text { if } d \leq d_{c} \\ L_{2 \mathrm{GR}}[d B], & \text { if } d \geq d_{c}\end{cases}$

$d_{c}=\frac{4 \pi h_{t} h_{r}}{\lambda}$

$L_{\log D}[d B]=L\left(d_{o}\right)+10 n \log _{10}\left(\frac{d}{d_{o}}\right)+X_{\sigma}$

On the other hand, RSS localization using radio maps/Wi-Fi fingerprints is carried out in two stages as shown in Fig. 1b: the offline/training phase and the real time/measurement phase. In the offline phase, the signal strength from the APs/anchors within the coverage area is measured (in different directions) at fixed points (known as reference points (RP)). These RPs are collated in form of a grid to cover the region of interest and is referred to as a radio map. In the measurement phase, the tracked device records the RSS of the APs/anchors present and carries out a comparison process with the offline database in order to estimate its location. In the literature, deterministic frameworks (such as nearest neighbor (NN) [28,29], $k$ - nearest neighbor $(k-\mathrm{NN})[28-30])$ or statistical methods/Bayesian inferences [31,32]) have been used to compare the raw data with the fingerprints. Some other works have adopted methods such as neural networks [33,34] or support vector machines (SVM) $[33,35,36]$. For these fingerprinting comparison methods, the $i$ th element in the radio map can be mathematically expressed as [37]:

$M_{i}=(B_{i}, \underbrace{\left\{\mathbf{a}_{i j} \mid j \in N_{i}\right\}}_{R_{i} \in R}, \theta_{i}), \quad i=1, \ldots, M$

where $B_{i}$ is the $i$ th cell with center $p_{i}, \boldsymbol{a}_{i j}$ is a vector of the RSS from $\mathrm{AP}_{j}, \theta_{i}$ contains the orientation in which the RSS was obtained: $\theta_{i}=O_{i} \in$ \{north, south, east, west $\}$ and $R_{i}$ is the $i$ th fingerprint for a set $R=\left\{R_{1}, \ldots, R_{M}\right\}$.

\subsubsection{Time-based}

In time-of-arrival based ranging, the distance is inferred using the propagation time of the radio wave between the transmitter/initiator and the receiver/responder. The time-of-flight (ToF) is given as the arrival time of the transmitted packet also known as time-of-arrival (ToA) minus the time-of-transmission (ToT) added by the initiator [25]. In order to obtain accurate estimations of the ToA, real-time synchronization of the clocks in the participating devices is required $[25,38]$. However in the literature, two-way ToA or hybrid methods using phase-shifts have been adopted to avoid synchronization $[39,40]$, as achieving real-time synchronization is challenging [38]. Provided that the coordinates of the anchors/APs are known, the T-R distance can be obtained by multiplying the ToF by the speed of the radio wave and the estimated distance is obtained in a similar way to Fig. 1a.

Rather than measuring the ToF, time differences between a tracked device and multiple anchors/APs can also be used as seen in time-difference-of-arrival (TDoA) schemes. TDoA can be implemented using the uplink channel to the anchors/AP (UTDoA) or the observed downlink channel from the AP (OTDoA). In OTDoA, hyperbolic multilateration is used to estimate the ToA from a master AP/anchor and two other participating APs. By subtracting the ToA of the slave APs from the master AP, the intersecting hyperbolas can be drawn. Given that the coordinates of the AP/anchors and the relative distances between all the nodes in the localization network are known, the estimated location/region of the tracked

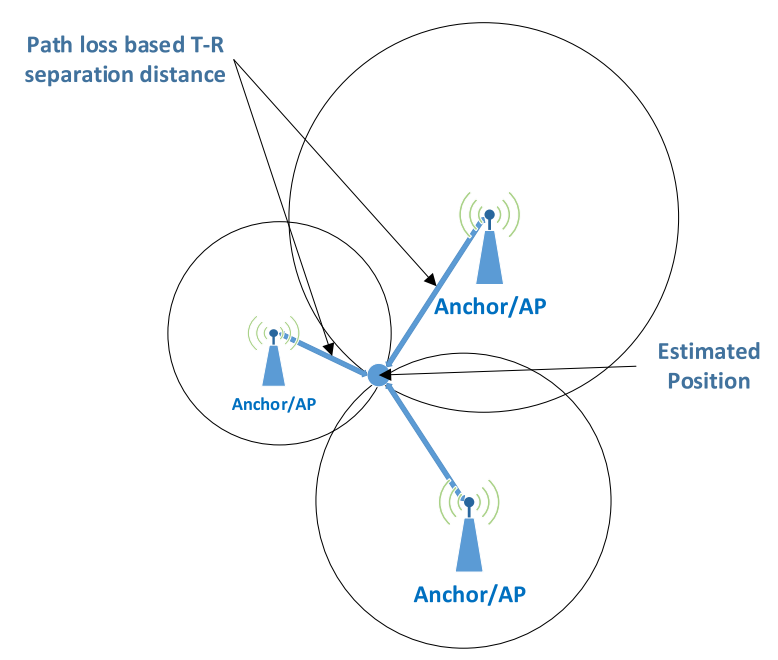

a)
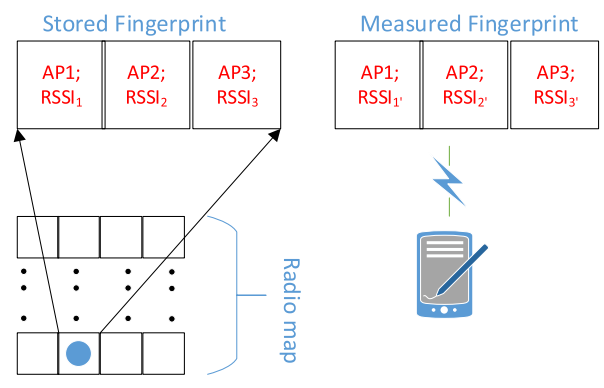

b)

Fig. 1. Illustration of a) $3 \mathrm{APs} / \mathrm{BSs}$ trilateration b) Radio map fingerprinting database. The fingerprint/database usually contains RSS samples of the APs surveyed or measured.

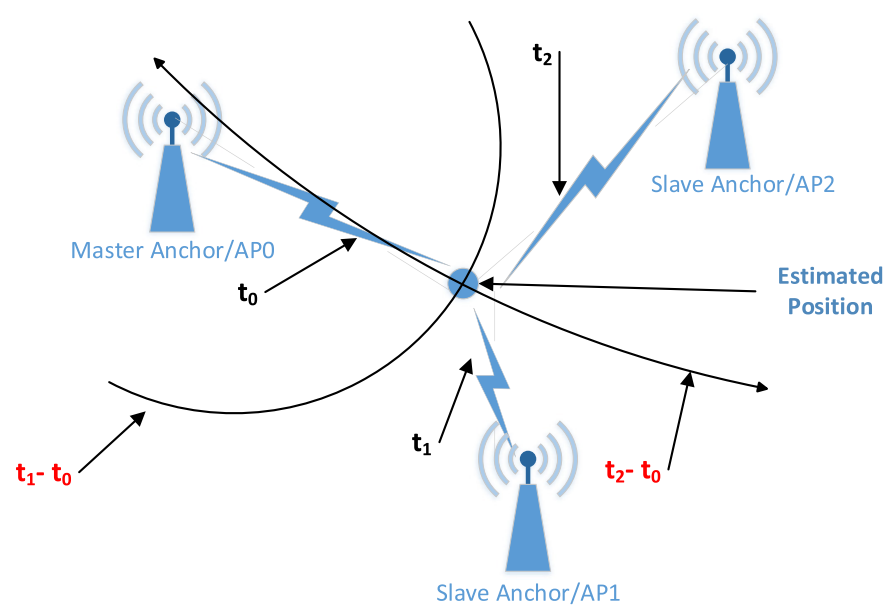

Fig. 2. Illustration of Observed Time Difference of Arrival (OTDoA).

device can be determined. This process is illustrated in Fig. 2. In general, delay-based ranging can be affected by uncertainties arising from clock drift, noise, multipath and relative motion [25].

\subsubsection{Angle-based}

In angle-of-arrival (AoA) distance measurements, the location of a tracked device is estimated using direction-finding methods and triangulation. Direction-finding in antenna systems can be implemented using a single directional antenna or by implementing an array. In the former, the antenna beam is steered and null or peak power detection schemes can be used to estimate the direction of the signal. Based on the assumption that phase shifted versions of 


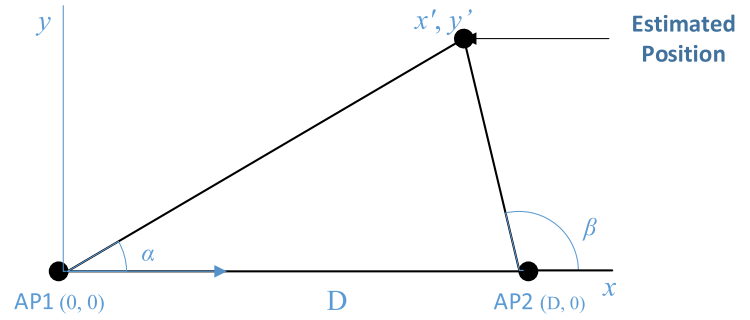

Fig. 3. Illustration of Angle of Arrival using two anchors/APs AP1 \& AP2.

the radio wave will impinge on the array, arrays such as a uniform linear array (ULA) can also be used to track direction of the radio wave sent from an anchor/AP. Some of the array methods include conventional beamforming and subspace techniques which rely on the properties of the array covariance matrix. In Fig. 3, AoA triangulation in 2-dimension using two anchors is illustrated and the coordinates of the tracked device can be estimated using (7), where $\alpha$ and $\beta$ are the arrival angles at the base station and $D$ is the distance between the base stations. Similar to time or RSS based techniques, uncertainties in estimation of the direction of the signal will create a region of possible coordinates of the device and the localization error can be calculated. From the triangulation depicted in Fig. 3, the localization error is optimized when the line joining the anchors and the device to the anchors forms an acute triangle [25].

$$
\begin{array}{r}
x^{\prime}=\frac{D \cdot \tan (\beta)}{\tan (\beta)-\tan (\alpha)} \\
y^{\prime}=\frac{D \cdot \tan (\alpha) D \cdot \tan (\beta)}{\tan (\beta)-\tan (\alpha)}
\end{array}
$$

\subsection{Localization performance criteria}

The performance of a positioning system can be characterized by the following metrics:

\subsubsection{Accuracy}

This characterizes the localization error between the ground truth and the estimated/predicted position. The root mean square error (RMSE) is widely used in the literature and can be obtained using (8), where $x_{p}$ and $y_{p}$ are the estimated coordinates/position and $x, y$ are the ground truth coordinates. The maximum/mean absolute position error or euclidean error can also be adopted.

$\mathrm{E}_{r r}=\sqrt{\left(x-x_{p}\right)^{2}+\left(y-y_{p}\right)^{2}}$

\subsubsection{Precision}

This is closely associated with the accuracy of a positioning system. It describes in percentage the likelihood of the reported accuracy. The accuracy and precision can be jointly reported using a cumulative distribution function (CDF) of the localization error.

\subsubsection{Availability}

This the amount of time a GNSS provides usable location information. It is usually expressed as a percentage of epochs for which the receiver's output meets the required specification.

\subsubsection{Continuity}

This criteria relates to the ability of the GNSS to operate without failure or interruption. It is usually reported as a probability that the receiver is operational after initialization.

\subsubsection{Integrity}

This parameter characterizes the correctness of the information supplied by the navigation system. In other words, it indicates the "trust" a user can have in the provided information. In addition, integrity exhibits the ability of a navigation system to provide the user with timely warnings when the information provided is inaccurate. The integrity of a navigation system can be reported via the alert limit (AL), integrity risk, time to alert (TTA) and protection level (PL) [41].

\subsubsection{Dilution of precision (DoP)}

This parameter characterizes the position boundary created by the transmitter-receiver geometry. Depending on the geometry created, the region of error or area representing the position uncertainty can be large or small. For example, if the tips of the receiversatellites unit vector lie in a plane, the DoP will be large and if they form a tetrahedron with large volume, the resulting DoP will be small [42]. The DoP can be expressed as position (PDoP), vertical (VDoP) or horizontal (HDoP) coordinates as well as time (TDoP).

Dependent on the application, some other performance indexes can also be used to evaluate a navigation system. Some of which include: time to first fix (TTFF), acquisition sensitivity, tracking sensitivity, reacquisition time, static and dynamic navigation accuracy and radio frequency interference such as jamming [43]. While the above performance criteria characterize GNSS receivers in general, the accuracy and precision is usually reported for localization frameworks.

\section{Wi-Fi based positioning}

\subsection{GNSS-free Wi-Fi localization systems}

Vehicle localization can be achieved without ranging estimation from an absolute positioning system such as GNSS. In this subsection, these systems are also referred to as "Standalone Wi-Fi based Localization". In theory, this can be implemented by using vehicleto-vehicle (V2V), vehicle-to-infrastructure (V2I)-based positioning or a mixture of both platforms. However, with respect to these Standalone systems, most implementations are based on either V2I (as discussed in this subsection) or as GNSS-free cooperative positioning (CP). With respect to relative positioning of CAVs, rather than ranging between a fixed infrastructure, the concept of $\mathrm{CP}$ using wireless ranging between vehicles in a peer-to-peer network configuration can also be adopted. ${ }^{2}$ In this subsection, the literature surveyed focuses on vehicle localization involving an anchor or a road side unit (RSU).

The authors in [39] simulated a single RSU localization framework for vehicles and were able to improve the location accuracy by fusing vehicle kinematics obtained from the on-board IMU. In addition, further improvements were obtained by imposing road constraints (in the $x$ direction), which was broadcasted by the RSU. In the simulation, the ego vehicle obtained its direction of travel from beacons sent by the RSU at the entry point to the motorway and the distance to the RSU was obtained by using two-way ToA. Depending on the estimated ranging distance, a threshold was imposed to select the type of Kalman filter adopted in fusing the sensor outputs. Based on the assumption that the distance from RSU to vehicle is usually much greater than the width of the road, lane boundaries were used to adjust the $x$-estimate obtained from the IMU. The simulated results showed that the average localization error (MMSE) resulting from the fusion framework was $1.8 \mathrm{~m}$ and the accuracy was improved by $60 \%$ when compared to a single RSU implementation ( $y$-direction).

\footnotetext{
2 The definition of CP is based on [44]. This review paper does not focus on GNSS free $\mathrm{CP}$, a description of $\mathrm{CP}$ systems for vehicular networks can be found in [45].
} 
A city wide measurement campaign was carried out in Singapore Jurong Lake District [46]; a geographical area with different urban scenarios. The measurement area (of 1.5 million acres) was divided into 4 sectors. The localization method adopted was based on fusing Wi-Fi RSSI/service set identifier (SSID) fingerprinting with vehicle kinetic motion obtained from an IMU. Thus, two radio maps were created for estimating the location of the vehicle in the offline phase. An EKF was used at the fusion stage and a $k-N N$ pattern matching was used with respect to the radio map. From the measurements carried out, it was observed that the rate of change of the RSSI varied within distances of $10 \mathrm{~m}$ to the AP. Intuitively, this variation was significantly reduced when the SSID only radio map was used. The localization error for the Wi-Fi + IMU system was less than $50 \mathrm{~m}$ for $60 \%$ of the time. In areas with good Wi-Fi coverage, the localization error reduced to $10 \mathrm{~m}$ using a SSID only radio map.

A dual RSU time based localization system was also presented in [47]. In the framework presented, the vehicle used two sets of beacon messages from two RSUs to estimate its location. In the first stage, the dual beacons were used to create two intersecting circles, a process similar to trilateration localization. The actual position of the vehicle was determined using the next set of beacon messages, whereby the angles between the movement vector and the road direction were compared and the location was determined from the two options created from the lateration stage. The authors also presented a mathematical model to provide coverage for any specified length and width of road. In addition, a beacon collision avoidance back off timer was introduced to minimize packet loss at the ego vehicle. Given that this avoidance mechanism might cause the beacons from a pair of RSUs to arrive at different times, the authors presented a position recalculation scheme to mitigate the effect of the time offset. For a straight road length of $2.5 \mathrm{~km}$ and width $12 \mathrm{~m}$, the localization error was in the range of $3 \mathrm{~m}$ and $5.5 \mathrm{~m}$ when one of the RSUs failed.

RSS measurements between target vehicles and four RSUs were also evaluated via simulation in [48]. In the simulation setup, the RSUs were placed at the corners of 4 corners of a square region (30 by 30). The RSS from each of the anchors was averaged and used to determine the location of the tracked vehicles. For a fixed ranging error (with $\sigma_{2}=0.25$ ), the localization error was approximately $0.16 \mathrm{~m}$. The accuracy remained relatively constant as the number of vehicles increased to about 100, with the accuracy increasing with decreasing ranging error.

Within typical inner city layouts with straight and curved paths, [49] adopted fusing TDoA ranging from a single RSU with IMU data and a digital map. The vehicle dynamics of the tracked vehicle was modeled using the velocity and arbitrary vehicle motion models. The former was used for straight paths and the latter for roads with bends. In order to bound the errors associated with the IMU, nonholonomic constraints [50] were adopted in estimating the body frame velocities. The heading observation obtained from a digital map and driving direction was used in the fusion framework. Using software defined radios (SDRs) in the tracked vehicle and RSU, quadratic interpolation was used to obtain higher resolution ToA data from the DSRC beacon frames. On the two test tracks used, the localization error was within 1-5 m. In addition, the authors reported that ToA ranging was sufficient for localization on straight paths with constant velocity.

By combining ToA ranging from multiple RSUs with IMU data, lane change detection, radar ranging and a high resolution digital map, the authors in [51] extended the work in [49] and were able to achieve localization errors of approximately $1 \mathrm{~m}$. Within the fusion scheme, models for the measurement data sources were created to feed into the fusion filter. Small scale measurements from the lane changes, digital map and car-borne radar were used to fine-tune the results obtained from the large scale measurement sources (RSU ranging and IMU data). The simulated scenario was a high/motor way with obstacles representing vehicles in other lanes. In addition, the EKF and PF were used to evaluate the performance of the fusion scheme. It was observed that the PF outperformed the EKF when vehicles in other lanes were close to the tracked vehicle.

Arrival angles of RSU beacons were used for wireless ranging in [52]. The AoA of the beacons were obtained using a ULA located on a tracked vehicle. Assuming the speed of the vehicle, variance and probability density function of the AoA were known, the initial estimate of the vehicle's location could be obtained from a maximum likelihood (ML) minimization problem. Subsequently, the location estimate at a given instant can be obtained using a kinematic model. A weighted least squares (WLS) algorithm was used instead of a ML due to the difficulty associated with the nonGaussian distribution of the noise and the requirement of the noise variance a priori. For a road segment $400 \mathrm{~m}$ long and $5 \mathrm{~m}$ wide, the AoA + WLS algorithm caused the localization error (when compared with a GPS receiver) to drop from an average of $8 \mathrm{~m}$ to approximately $3.5 \mathrm{~m}$. Nonetheless, the localization error was less than $1 \mathrm{~m}$ around the RSU.

With respect to hybrid wireless ranging, round trip time (RTT) and AoA wireless ranging from cooperative landmarks (or RSUs) were adopted in [53] for self localizing tracked vehicles. The ranging approach used RTT in the first phase to estimate the distance to the landmark and a smart antenna array to triangulate the response messages from the landmark. The wireless communication channel was designed using orthogonal pseudo random codes with slotted media access control (MAC) protocol, which ensured collisions were minimized. In the localization filter, distance and angle measurements to landmarks as well as tightly coupled vehicle odometry data (velocity and yaw rate) were used to obtain the global coordinates of the tracked vehicle. The scenario considered was an urban road crossing in Aschaffenburg, Germany with the cooperating landmarks at two diagonal corners. The average error recorded within a $80 \mathrm{~m}$ distance was $0.09 \mathrm{~m}$ and $0.28 \mathrm{~m}$ in $x$ and $y$-directions.

In order to reduce the measurement resources and effort required in creating a radio map, [54] used surface vector regression (SVR) to interpolate the RSS values while increasing the radio map resolution via an increased number of RPs. Other techniques adopted in the literature for developing the radio map include: spatial interpolation techniques such as inverse distance weighting (IDW) [55], Gaussian process regression [56,57], ray tracing [58], and parametric path loss models [28]. The fingerprinting localization module was tested via measurements in a University environment of $12000 \mathrm{~m}^{2}$. Within the test area, 175 APs were deployed and 30 RPs were used. In the test region, two tracks of $145 \mathrm{~m}$ and $135 \mathrm{~m}$ were used and the vehicle mean speed on both tracks was $4.86 \mathrm{~km} / \mathrm{hr}$ and $4.77 \mathrm{~km} / \mathrm{hr}$. By feeding the Wi-Fi fingerprinting localization results into a $\mathrm{KF}$, the mean error was reduced by $33.8 \%$ and $40.3 \%$ on both tracks. A summary of the literature discussed in this subsection is presented in Table 1.

\subsection{Hybrid Wi-Fi localization}

The literature presented in this subsection involves data filtering or fusion of GNSS pseudoranges with complementary sensor(s) presented in Section 1.

\subsection{1. $V 2 V$ based}

An assisted cooperative V2V communication network was presented in [59] using DSRC and GPS. The simulated framework considered three scenarios/environments: highway, tunnel and urban canyon at a fixed data fusion rate of $10 \mathrm{~Hz}$. In the respective environments, the positioning framework was investigated for 
Table 1

Summary of standalone Wi-Fi based localization methods.

\begin{tabular}{|c|c|c|c|c|c|}
\hline $\begin{array}{l}\text { Reference } \\
\text { (method) }\end{array}$ & Sensors adopted & Data fusion & Accuracy/Precision & Technique & Comments \\
\hline [39] ToA & $\begin{array}{l}\text { Accelerometer, } \\
\text { Gyroscopes } \\
\text { DSRC (802.11p) }\end{array}$ & Kalman filter & $\begin{array}{l}1.8 \mathrm{~m} \\
y \text {-direction } \\
\text { (MMSE) }\end{array}$ & Simulation & $\begin{array}{l}\text { Medium accuracy } \\
\text { High cost } \\
\text { Requires RSU at road } \\
\text { entry/exit \& mid points } \\
\text { Assumes RSU sends location } \\
\text { coordinates }\end{array}$ \\
\hline $\begin{array}{l}{[46]} \\
\text { RSS,SSID }\end{array}$ & $\begin{array}{l}\text { Accelerometer, } \\
\text { Gyroscopes } \\
\text { Wi-Fi Fingerprints }\end{array}$ & $\begin{array}{l}\text { Extended Kalman } \\
\text { filter }\end{array}$ & $\leq 50 \mathrm{~m}, 60 \%$ & Measurement & $\begin{array}{l}\text { Very low accuracy, Low cost } \\
\text { Low complexity }(k-N N) \\
\text { Opportunistic use of } \\
\text { available APs }\end{array}$ \\
\hline $\begin{array}{l}\text { [47] 2-way } \\
\text { reciprocal ToA }\end{array}$ & $\begin{array}{l}\text { Odometer } \\
\text { Compass }\end{array}$ & - & $3.3 \mathrm{~m}$ (RMSE) & Simulation & $\begin{array}{l}\text { Medium accuracy, High cost } \\
\text { Requires RSU to be placed } \\
\text { on mid points } \\
\text { Assumes RSU range covers } \\
\text { entire road width }\end{array}$ \\
\hline [48] RSS & - & - & $\begin{array}{l}0.18 \mathrm{~m}\left(\sigma^{2}=0.25\right) \\
(\mathrm{RMSE})\end{array}$ & Simulation & $\begin{array}{l}4 \text { RSUs required } \\
\text { Target vehicles are fixed }\end{array}$ \\
\hline [49], TDoA & $\begin{array}{l}\text { Accelerometer } \\
\text { Gyroscopes, } \\
\text { Digital Map }\end{array}$ & $\begin{array}{l}\text { Extended Kalman } \\
\text { filter }\end{array}$ & 1-5 m (RMSE) & Measurement & $\begin{array}{l}\text { Medium accuracy, } \\
\text { Medium complexity } \\
\text { Dedicated RSUs adopted, } \\
\text { High cost }\end{array}$ \\
\hline [51], ToA & $\begin{array}{l}\text { Accelerometer } \\
\text { Gyroscopes, } \\
\text { Radar, Digital Map }\end{array}$ & $\begin{array}{l}\text { Extended Kalman } \\
\text { filter } \\
\text { Particle filter }\end{array}$ & $1.02 \mathrm{~m}$ (RMSE) & Simulation & $\begin{array}{l}\text { High accuracy, cost \& } \\
\text { complexity } \\
\text { Lane change model is } \\
\text { assumed to be a } 1 \text { st order } \\
\text { Markov process }\end{array}$ \\
\hline [52], AoA & - & $\begin{array}{l}\text { Weighted least } \\
\text { squares }\end{array}$ & $3.5 \mathrm{~m}$ (RMSE) & Simulation & $\begin{array}{l}\text { Medium accuracy, High cost } \\
\text { High complexity }\end{array}$ \\
\hline [53] RTT, AoA & Odometer & $\begin{array}{l}\text { Extended Kalman } \\
\text { filter }\end{array}$ & $0.09 / 0.28 \mathrm{~m}(\mathrm{x} / \mathrm{y})$ & Measurement & $\begin{array}{l}\text { High accuracy, Medium } \\
\text { complexity } \\
\text { Dedicated RF-based } \\
\text { landmarks adopted }\end{array}$ \\
\hline $\begin{array}{l}{[54]} \\
\text { RSS fingerprinting } \\
\text { SVR }\end{array}$ & - & Kalman filter & $\begin{array}{l}6.18 \mathrm{~m} \text { (Track1) } \\
\leq 10 \mathrm{~m} 80 \% \\
\text { (Mean error) }\end{array}$ & Measurement & $\begin{array}{l}\text { Medium accuracy, Low } \\
\text { complexity } \\
\text { RSS samples of APs are } \\
\text { recorded at RPs }\end{array}$ \\
\hline
\end{tabular}

standalone GPS (non cooperative) and fused GPS + DSRC (with cooperative positioning), as well as a reduced data fusion rate. The authors aimed at addressing the spatial-time correlation in the noise models for fusion filters by replicating the simulations using a Gaussian independent and identically distributed (i.i.d.) noise processes and realistic noise models (such as 2-D GPS noise maps and 4-D V2V RSSI shadow maps). By introducing techniques such as differential measurements (DM) in the GPS data, RSSI noise correlation processes and reduced fusion rates, the fusion framework was able to show significant improvement with respect to noise correlation. The framework presented attained as much as $60 \%$ accuracy improvement in scenarios where the noise processes were correlated.

The $\mathrm{CP}$ radio-ranging based positioning system presented in [38] incorporates a RSSI based ranging module, GPS module (for initial location estimates), onboard sensor (for obtaining vehicle kinematics) and road maps (for enforcing latitudinal road constraints). The localization process in this work can be summarized into 3 phases: initialization, where the tracked vehicle obtains initial positioning information from a GNSS. Alternatively, this can be obtained from GPS data exchanged between participating vehicles or by trilateration of the inter vehicle distances; at the refinement stage, the estimates obtained in Phase 1 are updated; the last phase involves a recursive update of Phase 2 using the relative distances obtained. After carrying out ranging measurements within the cluster of participating vehicles, $n^{2}-n$ inter vehicle distance measurements and $n$ velocity profiles are stored at each vehicle. The road constraints adopted in this work is similar to [39], which ensured that the localization error was below the CramérRao lower bound (CRLB) and approximately $2.2 \mathrm{~m}$ during the simulation window.

Simulated vehicular mobility traces were used in [60] to evaluate a cooperative location sensor fusion algorithm. The proposed scheme adopted a reduced inertial sensor system (RISS) and RTT for radio ranging between cooperating vehicles. In the simulation model, erroneous GPS data (when available) was fused with the RISS using a loosely coupled KF for obtaining a rough estimate of the ranging distance. Thereafter, an EKF was used to fine-tune the estimate. The effect of increasing the number of participating vehicles with access to ideal GPS updates showed a reduction in the average RMSE. While an increased vehicle speed caused the localization accuracy to deteriorate, the errors were bounded to $0.3 \mathrm{~m}$ by the cooperative scheme during the simulation window. Intuitively, when realistic GPS updates (with errors) were used, the average localization error increased.

Two techniques known as consistency check (CC) [61] and double difference (DD) [62] were applied in [63] to mitigate the effects of multipath/NLOS signals in GNSS pseudoranges and V2V radio ranging. In the proposed algorithm, the first CC was applied to the raw measurements obtained from the GNSS receiver and a second applied to measurements obtained in the V2V-DD ranging. The output of both CC layers was then fed into a CP algorithm that estimated absolute and relative distances between participating vehicles and a reference vehicle. The simulation results show that for five participating vehicles, the mean localization error was less than or equal to $1 \mathrm{~m}$ when evaluating the relative distances. 
The authors also simulated a mixed scenario where some of the participating vehicles had access to multipath prone GNSS signals. In this scenario, the mean error ranged from $5.82 \mathrm{~m}$ to $7.00 \mathrm{~m}$. Nonetheless, the algorithm yielded higher accuracy when compared with a least squares (LS) CP scheme or when a single layer CC was adopted. In addition, when raw GNSS data was fed into the algorithm, the mean error increased to $9.68 \mathrm{~m}$.

Motion data and GPS location estimates were fused with ToA inter-vehicle ranging data using a $\mathrm{KF}$ in a multi-measurement approach presented in [64]. In the fusion setup, a multipath detection unit (MDU) and a localization enhancement unit (LEU) were included for detecting multipath effects in the estimate obtained from the KF and optimizing the neighbor information fed into the KF. Given that multipath propagation introduces noise in the pseudorange estimate, a feedforward backpropagation (FFB) algorithm was used to train an artificial neural network (ANN) for the purpose of minimizing the localization error. The algorithm for the LEU was based on selecting mobile anchors with the least estimated location uncertainty. The location uncertainty in this work was obtained from the variation in the time update provided by the KF and the GPS pseudorange. In the simulation setup, the vehicle traveled $5 \mathrm{~km}$ through open space and multipath regions. In comparison with $\mathrm{KF}$ based fusion, the algorithm reduced the localization mean error by $65 \%$ and $70 \%$ in the multipath regions encountered in the trajectory. Unlike the RSS and time based ranging methods presented, [65] used carrier frequency offset based range-rating; which is made up of the Doppler shift and clock drift between the ego and neighbor vehicles. The fusion framework used a Cubature Kalman filter (CKF) due to its simple structure and estimation performance. In order to make the fusion framework immune to the deviation between the usual Gaussian assumptions and the error probability density function, the $M$-estimator with the Huber technique was adopted. When compared to a standard or fixed constraint CKF, the adaptive restraint significantly reduced the upper bound of the RMSE.

\subsubsection{V2I (Anchor/RSU) based}

The RITS project in $[66,67]$ implemented and tested hybrid WiFi localization in different scenarios using a connected car designed for measurement campaigns. In [66], outdoor Wi-Fi RSS fingerprints were fused with laser-SLAM. The RSSI values entered into the Wi-Fi radio map in the online phase were normalized; where weak RSSIs were assigned 0 and strong RSSIs were assigned a value of 1 . In the offline phase, APs that weren't detected were assigned a value of -1 . In order to mitigate the effect of multipath fading, the authors implemented pure selection (2-antenna) spatial diversity at the receiver. A PF was adopted in fusing odometry data with GPS + SLAM or Wi-Fi + SLAM. The fusion method was able to select a suitable positioning method depending on the vehicle's environment, i.e. the hybrid method could be a fusion of GPS-SLAM or Wi-Fi-SLAM (when the GPS reference required for SLAM is not available). In the trials carried out, the vehicle path had areas with poor and good Wi-Fi/GPS coverage. The average/maximum localization error from the fusion framework presented was $2.7 / 8 \mathrm{~m}$. This fusion framework was extended to car parks in [67] where the typical speed of the car was about $10-12 \mathrm{~km} / \mathrm{hr}$. A mathematical linear model was presented for interpolating the vehicle's location with respect to the latency associated with estimating the location. The latency arises from the frequency of the Wi-Fi scans and the vehicle speed. Over a $1 \mathrm{~km}$ path, the localization error was $2.25 \mathrm{~m}$.

A fusion framework that optimally combines vehicle location information obtained from 802.11p, BeiDou navigation satellite system (BDS), GPS, vehicle on-board diagnostic (OBD) and an INS was presented in [68]. The main focus of the paper was on a dual threshold ToA ranging of OFDM preambles from an IEEE 802.11p
AP. The time related information was taken from 10 short preambles in the 802.11p PHY and vehicle distance was inferred by multiplying this value with the speed of the radio wave. Location estimation accuracy was improved by using differential location information obtained from BDS and GPS, as well as fusing vehicle diagnostics from a gyroscope based IMU. The fusion scheme presented used a PF to fuse BDS, GPS and 802.11p location estimates. Simultaneously, OBD was fused with INS data using a Sage-Husa filter. The overall estimate of the vehicle location was obtained by feeding the fused data from the sub filters into a KF.

Two RSUs were used to reduce the location error in [69]. The selected area for obtaining and testing the vehicle trace was a dense taxi area of $6 \mathrm{~km}^{2}$ in Rome, Italy. In the offline phase of the proposed solution, a road map made up of edges (or road constraints) was used to create a mobility model for the simulation. Using a selected number of nodes (with known locations) and road side edges, the algorithm within the RSU calculates and forwards the estimation error between the RSU and vehicle based on its erroneous GNSS location estimate. Within the test regions of $6 \mathrm{~km}^{2}$ and $1.8 \mathrm{~km}^{2}$, the RSU localization framework reduced the average location error from $6.83 \mathrm{~m} / 7.21 \mathrm{~m}$ to $2.78 \mathrm{~m} / 0.74 \mathrm{~m}$.

The architecture presented in [55] obtained vehicle position by using two schemes. The first method involved fusing GPS pseudoranges with vehicle kinematics via a $\mathrm{KF}$ and the second method fused vehicle kinematics with Wi-Fi wireless ranging using a PF. A two-stage integration block was used to combine the Wi-Fi based localization with GPS data in the second method. In order to reduce the effect of fading in the fingerprinting training phase, the RSS measurements were time averaged and IDW interpolation was used to create the radio map. In the measurement phase, localization was achieved using NN. In the framework presented, the Wi-Fi/GPS integration module detects uncertainty in the GPS pseudorange by inspecting the DoP and checks for inconsistencies in the KF update and the GPS pseudorange. In scenarios where the GPS position data was uncertain, a residual weighting function was used to combine both filter outputs. Within a square shaped commercial area of $56000 \mathrm{~m}^{2}$ in Schenzhen, China, the average RMSE of the proposed framework was $11.83 \mathrm{~m}$.

Similar to [55], dead reckoning (DR) was included in [70] in a different fusion framework. The framework presented used a federated unscented Kalman filter (UKF) to fuse GPS and DR data; a $\mathrm{KF}$ to fuse GPS with Wi-Fi and an information sharing filter to optimally combine the outputs of the KF and UKF. Between the Wi-Fi + GPS KF and the master filter, a multipath detection module was included for inspecting the wireless ranging data obtained. The fusion method was tested in a simulation scenario covering $5 \mathrm{~km}$ with typical urban artifacts that resulted in GNSS signal blockage and multipath propagation for a portion of the trajectory. The reported localization error of the integrated framework was $5.7 / 5.8 \mathrm{~m}(x / y)$. In addition, the authors observed that the accuracy obtainable from the Wi-Fi fingerprinting was dependent on the vehicle speed.

Rather than the typical usage of GPS pseudorange and one or two other complementary positioning sensors, the integrated localization framework presented in [71] evaluated position data from a wide variety of sources which include GPS, RFID, V2V, V2I and DR. In the integrated framework, the tracked vehicles have connections to other vehicles, RSUs and GPS satellites. Three different models were used to obtain the position of a tracked vehicle. The first model estimated the distance between a vehicle and a unit (RSU/GPS satellite/vehicle), the second modeled the distance between vehicle and a RFID reader and the third represented the vehicle's state transition using DR. These models were combined into a nonlinear optimization problem. In order to reduce the latency in obtaining the position estimate, the algorithm used a CRLB to filter out redundant connections or unreliable sensor data. The 
integrated algorithm was tested via simulations using a road network made up of highways, commercial, residential, forest, and tunnel/parking areas. In clear, dense and semi-dense areas, the localization error for GPS or any of the hybrid systems was less than $15 \mathrm{~m}$ and exceeded $25 \mathrm{~m}$ within a tunnel. Within the tunnel, the integrated framework reduced the localization error to less than $5 \mathrm{~m}$. Without DR, the integrated framework was able to reduce the localization error by $80 \%$.

With respect to multi-GNSS, [72] used two GNSS receivers separated longitudinally on an ego vehicle. The localization system used RTT ranging to a RSU, with an antenna station capable of sending corrections for real-time kinematic (RTK) positioning. Within the framework, DD was first applied to the data obtained from the ego vehicle's secondary antenna and a cooperating vehicle or RSU and later applied to the ego vehicle's main antenna and master station. The framework was tested using a straight path of $1 \mathrm{~km}$ with trees and buildings included along the traveled path. Over the test track, the localization error was less than $0.8 \mathrm{~m}$.

\subsubsection{Mixed V2V and V2I}

[73] presented a simulation of AoA-based cooperative localization using IEEE 802.11p. The simulation considered V2V, V2I and a mixture of both vehicular communication schemes. Periodic beacons (sent by a RSU or a specialized vehicle) were used to initiate the Kalman filtering process at vehicles able to execute the location tracking scheme. In the simulation setup, these special vehicles were regarded as U-vehicles, which were also capable of broadcasting GPS and INS information via V2V communications. The location estimation filter was used to estimate velocity and position in both $x$ and $y$ directions. In a V2I only scenario, the filter performed better in the transverse direction and the localization (velocity and position) errors reduced as the U-vehicle approached the RSU. At the RSU crossing, the errors dropped below $1 \mathrm{~m}$ and steadily increased as the vehicle moved further away. Intuitively, the localization errors reduced as the RSU beacon rate increased. The authors also extended the simulation to a V2V scenario where the proposed framework was able to reduce the initial localization error by as much as 30\% when the vehicles crossed. The error was within $1 \mathrm{~m}$, when the tracked vehicle had a high localization accuracy. From the V2V simulations, it was discovered that 3-4 cars were sufficient, as subsequent additions did not yield corresponding improvements in the localization accuracy of the U-vehicle. In comparison with [38], this framework achieves similar localization accuracy by using a lesser number of vehicles. When the V2V setup was simulated alongside an active RSU, the localization accuracy improved by at least $30 \%$ when compared to the framework's vehicle-to-road side unit (V2RSU) implementation.

The localization setup presented in [74] estimated 2D/3D position and velocity (heading and speed) of a tracked vehicle by fusing map information, on-board sensors (INS, V2V ranging), GPS pseudoranges and RSU ranging (when available). A parametric path loss model was used to estimate inter-vehicle distance and road segments were defined on a map using two edge points. After obtaining the posterior function, a maximum a posteriori estimate was used to obtain the posterior density. The main positioning PF was responsible for predicting velocity and heading, and the secondary filter fed fused INS data obtained from smartphones into the PF. For the entire trajectory, the RMSE reduced by $3 \%$ when the GPS + V2V + Map was compared with erroneous GPS pseudoranges. In scenarios along the trajectory where the participating vehicles in the V2V ranging were close (or within $40 \mathrm{~m}$ ), the framework introduced a $28 \%$ improvement in the RMSE. Given the positioning errors reported, the authors also noted that the RMSE could be further reduced by adopting lateral road map restrictions as evident in $[38,39,69]$. In Table 2 , a summary of the literature discussed is presented.

\section{Conclusion}

This paper has presented recent implementations of AP based Wi-Fi enabled vehicle localization using Wi-Fi only ranging and fusion with GNSS or complementary positioning systems. The surveyed literature shows that the use of anchor based Wi-Fi localization requires additional investigation from a research, measurement and testing perspective. While wireless ranging is widely adopted in Wi-Fi based localization, it is expected that weather conditions such as rain attenuation will introduce additional errors in location estimation. However, if these APs are affected in a similar manner, the effect on the position estimates could be negligible $[75,76]$. Although, this survey paper has focused on WiFi, GNSS updates can be fused with other complimentary location sensors [77-80]. Moreover, multiple wireless ranging techniques discussed can be combined as shown in [81].

Most of the localization errors presented from the literature do not meet the current requirements for CAVs in ITS, it is evident that these techniques and technologies can be further developed to improve location accuracy and precision. In addition, $70 \%$ of the surveyed literature are based on simulations which have inherent limitations. It is essential that the algorithms proposed are tested in real life experimental scenarios. From a vehicle-to-infrastructure perspective, some of the viable areas open for research include:

1) Layer 1 fusion of RSU signals: Vehicle positioning can be improved by applying data fusion at the PHY layer of the RSU. By introducing GNSS features present in pseudolites, RSUs can be modified at the PHY layer to transmit fused beacons/ranging frames made up of GNSS and 802.11p wireless access in vehicular environments (WAVE) signals. The performance of this fusion technique can be rapidly evaluated and tested by using testbeds designed from software defined radios (SDRs). With SDR, RF signals with Wi-Fi and GNSS protocols can be emitted in a mixed mode whereby transmitted signals can be controlled with respect to time, phase and a predefined fading model. In addition, this approach can be combined with geophysical models which can be used as an iterative mechanism for evaluating how artificial RF signals mimic radio propagation as well as its effects.

2) $5 \mathrm{G}$ millimeter wave (mmWave): Rather than current sub-6 $\mathrm{GHz}$ implementations, it is envisaged that $5 \mathrm{G}$ device-to-device (D2D) communications will adopt radio interfaces such as New Radio (NR) as well as new 5G waveforms and multiple access techniques at millimeter wave frequencies. By using highly directional antennas with narrow beams, millimeter wave radio maps for localization fingerprinting can be developed. Moreover, 5G mmWave can be used to exploit multipath propagation within and around the vehicle by using techniques such as beamforming to improve the signal reception in dense V2X networks. In a typical V2X communication network, this technology can also be extended to ToA, AoA or RSS path loss based wireless ranging for CAV positioning. Furthermore, the high bandwidth available in $5 \mathrm{G}$ mmWave can be also exploited for new services and products relating to delivering HD content and in-car infotainment.

3) Dual RSU radio interface: With IEEE 802.11p WAVE ahead of $\mathrm{C}-\mathrm{V} 2 \mathrm{X}$, wireless ranging errors can be reduced by integrating both technologies within the RSU and OBU. Given that both technologies implement PHY and MAC layers relatively differently, it is envisaged that the ranging performance will vary due to intrinsic characteristics of the radio and core networks. In scenarios whereby a singular specific ranging method (delay, RSS, angle, range rating) is implemented, positioning data from dual ranging using both (WAVE + C-V2X) platforms can be optimally filtered to improve the location estimate. More- 
Table 2

Summary of hybrid Wi-Fi localization methods.

\begin{tabular}{|c|c|c|c|c|c|}
\hline $\begin{array}{l}\text { Method } \\
\text { (Reference) }\end{array}$ & Sensors adopted & Data fusion & Accuracy/Precision & Technique & Comments \\
\hline $\begin{array}{l}\text { [38]ToA, RSS } \\
\text { Road map }\end{array}$ & $\begin{array}{l}\text { GPS } \\
\text { Onboard sensors }\end{array}$ & $\begin{array}{l}\text { Extended Kalman } \\
\text { filter }\end{array}$ & $2.2 \mathrm{~m}$ (RMSE) & Simulation & $\begin{array}{l}\text { Medium accuracy, High cost } \\
\text { Medium complexity, } \\
\text { Requires initial Vehicle } \\
\text { position from GNSS }\end{array}$ \\
\hline $\begin{array}{l}{[66] \text { ToA, RSS }} \\
\text { Odometry data }\end{array}$ & $\begin{array}{l}\text { GPS, Laser-SLAM, } \\
\text { Wi-Fi Fingerprints }\end{array}$ & $\begin{array}{l}\text { Particle filter \& } \\
\text { Neural network }\end{array}$ & $\begin{array}{l}2.7 \mathrm{~m} \\
\text { (Euclidean error) }\end{array}$ & Measurement & $\begin{array}{l}\text { Medium accuracy, High cost } \\
\text { Medium complexity, RSS } \\
\text { samples of APs are recorded } \\
\text { at RPs }\end{array}$ \\
\hline $\begin{array}{l}\text { [55] ToA, } \\
\text { RSS Fingerprinting }\end{array}$ & - & $\begin{array}{l}\text { Kalman filter } \\
\text { Particle filter }\end{array}$ & $\begin{array}{l}11.83 \mathrm{~m}, \leq 10 \mathrm{~m} \\
60 \% \\
\text { (RMSE) }\end{array}$ & Measurement & $\begin{array}{l}\text { Low accuracy, High cost } \\
\text { Low complexity, RSS } \\
\text { samples of APs are recorded } \\
\text { at RPs }\end{array}$ \\
\hline $\begin{array}{l}\text { [59] ToA, RSS } \\
\text { (urban canyon } \\
\text { correlated } \\
\text { noise \& } \\
\text { shadowing) }\end{array}$ & GPS, DSRC & Particle filter & $\begin{array}{l}\leq 4 \mathrm{~m}, 99 \% \\
\leq 1 \mathrm{~m}, 70 \% \\
\mathrm{MAE}\end{array}$ & Simulation & $\begin{array}{l}\text { High accuracy } \\
\text { Robust to correlated DSRC } \\
\text { shadowing and GPS noise }\end{array}$ \\
\hline [60] RTT & $\begin{array}{l}\text { GPS } \\
\text { Gyroscope, } \\
\text { Odometer }\end{array}$ & $\begin{array}{l}\text { Kalman filter, } \\
\text { Extended Kalman } \\
\text { filter }\end{array}$ & $\begin{array}{l}\leq 0.2 \mathrm{~m} \text { (ideal GPS) } \\
\leq 1.3 \mathrm{~m} \\
(\mathrm{RMSE})\end{array}$ & Simulation & $\begin{array}{l}\text { Very high accuracy (ideal } \\
\text { GPS) } \\
\text { High complexity }\end{array}$ \\
\hline $\begin{array}{l}\text { [63] ToA, } \\
\text { Double difference }\end{array}$ & GNSS & $\begin{array}{l}\text { Weighted } \\
\text { cooperative } \\
\text { localization }\end{array}$ & $\begin{array}{l}1 \mathrm{~m} \text { (Relative) } \\
5.82-7.00 \mathrm{~m} \\
\text { (Absolute) } \\
\text { (Mean error) }\end{array}$ & Simulation & $\begin{array}{l}\text { Medium accuracy } \\
\text { Medium complexity } \\
\text { High cost }\end{array}$ \\
\hline $\begin{array}{l}{[64] \text { ToA }} \\
\text { (V2V \& GPS) }\end{array}$ & $\begin{array}{l}\text { GPS } \\
\text { INS, VANET }\end{array}$ & $\begin{array}{l}\text { Kalman filter } \\
\text { Neural network }\end{array}$ & $\begin{array}{l}3.87 \mathrm{~m}, \leq 5 \mathrm{~m} 82 \% \\
\text { (Mean error) }\end{array}$ & Simulation & $\begin{array}{l}\text { Moderate accuracy, robust } \\
\text { Medium complexity, High } \\
\text { cost }\end{array}$ \\
\hline $\begin{array}{l}{[65] \text { ToA }} \\
\text { CFO range-rating }\end{array}$ & $\begin{array}{l}\text { GNSS } \\
\text { DSRC }\end{array}$ & $\begin{array}{l}\text { Cubature Kalman } \\
\text { filter } \\
\text { Huber technique } \\
\text { M-estimator }\end{array}$ & $\begin{array}{l}9.38 \mathrm{~m} \text { (east) } \\
10.6 \mathrm{~m} \text { (north) } \\
\text { (Max mean error) }\end{array}$ & Simulation & $\begin{array}{l}\text { Medium accuracy } \\
\text { Robust, } \\
\text { High complexity }\end{array}$ \\
\hline $\begin{array}{l}\text { [67] ToA, RSS } \\
\text { odometry data }\end{array}$ & $\begin{array}{l}\text { GPS, laser-SLAM, } \\
\text { Wi-Fi Fingerprints }\end{array}$ & $\begin{array}{l}\text { Particle filter \& } \\
\text { Neural network }\end{array}$ & $\begin{array}{l}2.80 \mathrm{~m} \text { (RMSE) } \\
2.25 \mathrm{~m} \text { (Euclidean } \\
\text { error) }\end{array}$ & Measurement & $\begin{array}{l}\text { Medium accuracy } \\
\text { High cost \& Medium } \\
\text { complexity } \\
\text { RSS samples of APs are } \\
\text { recorded at RPs }\end{array}$ \\
\hline [68] ToA & $\begin{array}{l}\text { BDS, GPS, } \\
\text { Gyroscope }\end{array}$ & $\begin{array}{l}\text { Kalman filter, } \\
\text { Sage-Husa filter, } \\
\text { Particle filter }\end{array}$ & - & Simulation & $\begin{array}{l}\text { High complexity } \\
\text { High cost }\end{array}$ \\
\hline $\begin{array}{l}\text { [69] ToA, } \\
\text { Road map }\end{array}$ & WAVE & - & $\begin{array}{l}0.74 \mathrm{~m}\left(1.8 \mathrm{~km}^{2}\right) \\
2.78 \mathrm{~m}\left(6.0 \mathrm{~km}^{2}\right)\end{array}$ & Simulation & $\begin{array}{l}\text { High accuracy, High cost } \\
\text { Low complexity }\end{array}$ \\
\hline $\begin{array}{l}\text { [70] ToA, } \\
\text { RSS Fingerprinting }\end{array}$ & $\begin{array}{l}\text { Gyroscope } \\
\text { Odometer }\end{array}$ & $\begin{array}{l}\text { Kalman filter } \\
\text { Unscented } \\
\text { Kalman Filter }\end{array}$ & $\begin{array}{l}5.7 \mathrm{~m} / 5.8 \mathrm{~m}(\mathrm{x} / \mathrm{y}) \\
\leq 10 \mathrm{~m} 100 \% \\
\text { (RMSE) }\end{array}$ & Simulation & $\begin{array}{l}\text { Low accuracy } \\
\text { Low complexity } \\
\text { High cost }\end{array}$ \\
\hline $\begin{array}{l}\text { [71], ToA } \\
\text { (V2I, V2V, GPS) }\end{array}$ & RFID & $\begin{array}{l}\text { Maximum a } \\
\text { posteriori (MAP) } \\
\text { estimation }\end{array}$ & $\begin{array}{l}2.94 \mathrm{~m}, \leq 3.37 \mathrm{~m} \\
80 \% \\
\text { framework+DR } \\
\text { (RMSE) }\end{array}$ & Simulation & $\begin{array}{l}\text { Medium accuracy, } \\
\text { Highly computational } \\
\text { High cost }\end{array}$ \\
\hline $\begin{array}{l}\text { [72], RTT } \\
\text { Double difference } \\
\text { (RTK) }\end{array}$ & $\begin{array}{l}\text { GPS RTK, } \\
\text { DSRC }\end{array}$ & - & 0.698 m (RMSE) & Measurement & $\begin{array}{l}\text { High accuracy } \\
\text { High complexity, High cost } \\
\text { Dedicated RSUs adopted }\end{array}$ \\
\hline [73], AoA & Gyroscope & Kalman filter & $\leq 1 \mathrm{~m}(\mathrm{RMSE})$ & Simulation & $\begin{array}{l}\text { High accuracy at crossing } \\
\text { High complexity, High cost }\end{array}$ \\
\hline $\begin{array}{l}{[74] \text { ToA, RSS }} \\
\text { Road map }\end{array}$ & $\begin{array}{l}\text { Gyroscope } \\
\text { Accelerometer } \\
\text { Magnetometer } \\
\text { DSRC }\end{array}$ & $\begin{array}{l}\text { Unscented } \\
\text { Kalman filter } \\
\text { (UKF) } \\
\text { Particle filter }\end{array}$ & $\begin{array}{l}9.47 \mathrm{~m}, \leq 11.7 \mathrm{~m} \\
70 \% \\
(\mathrm{RMSE})\end{array}$ & Measurement & $\begin{array}{l}\text { Robust to failure, High cost } \\
\text { High complexity, Vehicles } \\
\text { used as mobile anchors } \\
\text { Scalable (distributed } \\
\text { architecture) }\end{array}$ \\
\hline
\end{tabular}

over, this can be also extended to hybrid dual ranging techniques, whereby at two ranging methods in both WAVE and C-V2X are used to obtain positioning data for a CAV traveling through a multipath/NLOS environment. Typically a CAV will experience different propagation effects as it travels on any given trajectory, it is envisaged that these radio ranging methods from both WAVE and C-V2X will experience differ- ent propagation characteristics and consequently have varying performance.

4) Antenna beam steering for V2X: With millimeter wave communications being adopted in DSRC/WAVE implementations, signal processing techniques such as beam steering can be applied to the antenna interface to improve wireless ranging between the RSU and the OBU. With adaptive beam steering, 
LoS links can be maintained to CAVs with increased mobility. While this technique offers an avenue for rapid uptake of mmWave communication in ITS, the challenge of Doppler spread, beam steering overhead and phased-array response needs to be investigated further.

5) Millimeter wave (mmWave) Radar: By adopting millimeter wave radar at $29 / 79 \mathrm{GHz}$, the positioning of CAVs can be improved using short range radars (SRR) as well as Radar maps. A typical use case scenario for mmWave radar is an intersection surveillance systems. With the use of radar modules (with range-resolution capable of detecting pedestrians), objects in the wireless channel can be detected in real time with timely alerts delivered to surrounding vehicles. In order to obtain high distance resolution using this technique, the bandwidth of the pulses also needs to be investigated.

Currently, perception based techniques are ahead of wireless-based localization, nonetheless all the technologies discussed have individual pros and cons. It is envisaged that a cooperative infrastructure that encompasses a wide variety of techniques would create a scenario where the localization errors of CAVs and objects within and around the vehicle is reduced to acceptable ITS limits. With the deployment of carrier grade/enterprise Wi-Fi and small cells rapidly increasing in urban areas, it is envisaged that CAVs will be able to make use of these short ranged radio access networks for not only traffic management and driver assistance applications but for localization with high accuracy.

\section{Declaration of competing interest}

The authors declare that they have no known competing financial interests or personal relationships that could have appeared to influence the work reported in this paper.

\section{References}

[1] European Commission, Intelligent transport systems [Online]. Available: https://ec.europa.eu/transport/themes/its_en.

[2] D. Gingras, An Overview of Positioning and Data Fusion Techniques Applied to Land Vehicle Navigation Systems, Automotive Informatics and Communicative Systems: Principles in Vehicular Networks and Data Exchange, Information Science Reference, 2009 [Online]. Available: https://books.google.co.uk/books?id= ioVCDQEACAAJ.

[3] K. Jo, J. Kim, D. Kim, C. Jang, M. Sunwoo, Development of autonomous car-Part i: distributed system architecture and development process, IEEE Trans. Ind. Electron. 61 (12) (Dec 2014) 7131-7140.

[4] M. Schreiber, H. Königshof, A. Hellmund, C. Stiller, Vehicle localization with tightly coupled GNSS and visual odometry, in: IEEE Intelligent Vehicles Symposium (IV), June 2016, pp. 858-863.

[5] C. Li, B. Dai, T. Wu, Vision-based precision vehicle localization in urban environments, in: 2013 Chinese Automation Congress, Nov 2013, pp. 599-604.

[6] E. Ward, J. Folkesson, Vehicle localization with low cost radar sensors, in: IEEE Intelligent Vehicles Symposium (IV), June 2016, pp. 864-870.

[7] H.V. Dung, T.M. Chi, N.T. Nga, L.D. Truong, H.D. Trung, A vehicle positioning and navigation system design using mini2440 arm and Linux, in: 2013 Third World Congress on Information and Communication Technologies, WICT 2013, Dec 2013, pp. 74-78.

[8] X. Cui, T.A. Gulliver, J. Li, H. Zhang, Vehicle positioning using 5G millimeterwave systems, IEEE Access 4 (2016) 6964-6973.

[9] M. Driusso, C. Marshall, M. Sabathy, F. Knutti, H. Mathis, F. Babich, Vehicular position tracking using LTE signals, IEEE Trans. Veh. Technol. 66 (4) (April 2017) 3376-3391.

[10] J. Georgy, T. Karamat, U. Iqbal, A. Noureldin, Enhanced MEMS-IMU/odometer/GPS integration using mixture particle filter, GPS Solut. 15 (3) (Jul 2011) 239-252 [Online]. Available: https://doi.org/10.1007/s10291-010-0186-4.

[11] L. Ojeda, J. Borenstein, Personal dead-reckoning system for GPS-denied environments, in: 2007 IEEE International Workshop on Safety, Security and Rescue Robotics, Sept 2007, pp. 1-6.

[12] L. Andreone, R. Brignolo, S. Damiani, G.V. Fulvio Sommariva, S. Marco, SAFESPOTintegrated project: final report - public version [Online]. Available: http://www.safespot-eu.org/.
[13] N. Mattern, G. Wanielik, Camera-based vehicle localization at intersections using detailed digital maps, in: IEEE/ION Position, Location and Navigation Symposium, May 2010, pp. 1100-1107.

[14] 5G-PPP, 5G automotive vision [Online]. Available: https://5g-ppp.eu/.

[15] IEEE standard for information technology- local and metropolitan area networks- specific requirements- part 11: wireless lan medium access control (MAC) and physical layer (PHY) specifications amendment 6: wireless access in vehicular environments, in: IEEE Std 802.11p-2010 (Amendment to IEEE Std 802.11-2007 as Amended by IEEE Std 802.11k-2008, IEEE Std 802.11r-2008, IEEE Std 802.11y-2008, IEEE Std 802.11n-2009, and IEEE Std 802.11w-2009), July 2010, pp. 1-51.

[16] ETSI, Intelligent transport systems (ITS): access layer specification for intelligent transport systems operating in the $5 \mathrm{GHz}$ frequency band. [Online]. Available: https://www.etsi.org.

[17] IEEE standard for wireless access in vehicular environments (WAVE) - multichannel operation - redline, in: IEEE Std 1609.4-2016 (Revision of IEEE Std 1609.4-2010) - Redline, March 2016, pp. 1-206.

[18] I. Skog, P. Handel, In-car positioning and navigation technologies-a survey IEEE Trans. Intell. Transp. Syst. 10 (1) (March 2009) 4-21.

[19] S. Kuutti, S. Fallah, K. Katsaros, M. Dianati, F. Mccullough, A. Mouzakitis, A survey of the state-of-the-art localization techniques and their potentials for autonomous vehicle applications, IEEE Int. Things J. 5 (2) (April 2018) 829-846.

[20] R.M. Sandoval, A.J. Garcia-Sanchez, J. Garcia-Haro, Improving RSSI-based pathloss models accuracy for critical infrastructures: a smart grid substation casestudy, IEEE Trans. Ind. Inform. 14 (5) (May 2018) 2230-2240.

[21] K. Shamaei, J. Khalife, Z.M. Kassas, A joint TOA and DOA approach for positioning with LTE signals, in: 2018 IEEE/ION Position, Location and Navigation Symposium, PLANS, April 2018, pp. 81-91.

[22] T. Rappaport, Wireless Communications: Principles and Practice, 2nd ed., Prentice Hall PTR, Upper Saddle River, NJ, USA, 2001.

[23] A. Goldsmith, Wireless Communications, Cambridge University Press, 2005 [Online]. Available: https://books.google.co.uk/books?id=n-3ZZ9i0s-cC.

[24] J.-H. Kim, W.-Y. Yeo, A coherent data filtering method for large scale RF fingerprint Wi-Fi positioning systems, EURASIP J. Wirel. Commun. Netw. 2014 (1) (Jan 2014) 13 [Online]. Available: https://doi.org/10.1186/1687-1499-2014-13.

[25] A. Bensky, Wireless Positioning Technologies and Applications, Artech House, Inc., Norwood, MA, USA, 2007.

[26] T.O. Olasupo, C.E. Otero, L.D. Otero, K.O. Olasupo, I. Kostanic, Path loss models for low-power, low-data rate sensor nodes for smart car parking systems, IEEE Trans. Intell. Transp. Syst. 19 (6) (June 2018) 1774-1783.

[27] M. Cheffena, M. Mohamed, Empirical path loss models for wireless sensor network deployment in snowy environments, IEEE Antennas Wirel. Propag. Lett. 16 (2017) 2877-2880.

[28] S. Yiu, M. Dashti, H. Claussen, F. Perez-Cruz, Wireless RSSI fingerprinting localization, Signal Process. 131 (2017) 235-244 [Online]. Available: http:// www.sciencedirect.com/science/article/pii/S0165168416301566.

[29] B. Li, J. Salter, A.G. Dempster, C. Rizos, Indoor positioning techniques based on wireless LAN, in: The 1st International Conference on Wireless Broadband and Ultra Wideband Communications, 2007, pp. 13-16.

[30] B.J. Mohd, I. Amro, A. Alhasani, Indoor Wi-Fi tracking system using fingerprinting and Kalman filter, in: IEEE Jordan Conference on Applied Electrical Engineering and Computing Technologies, AEECT, Oct 2017, pp. 1-6.

[31] S. Ito, N. Kawaguchi, Bayesian based location estimation system using wireless lan, in: Third IEEE International Conference on Pervasive Computing and Communications Workshops, March 2005, pp. 273-278.

[32] N. Pritt, Indoor location with Wi-Fi fingerprinting, in: 2013 IEEE Applied Imagery Pattern Recognition Workshop, AIPR, Oct 2013, pp. 1-8.

[33] Y. Basiouny, M. Arafa, A.M. Sarhan, Enhancing Wi-Fi fingerprinting for indoor positioning system using single multiplicative neuron and PCA algorithm, in: 12th International Conference on Computer Engineering and Systems, ICCES, Dec 2017, pp. 295-305

[34] L.A. Castro, J. Favela, Reducing the uncertainty on location estimation of mobile users to support hospital work, IEEE Trans. Syst. Man Cybern., Part C, Appl. Rev. 38 (6) (Nov 2008) 861-866.

[35] R. Zhou, J. Chen, X. Lu, J. Wu, CSI fingerprinting with SVM regression to achieve device-free passive localization, in: 2017 IEEE 18th International Symposium on a World of Wireless, Mobile and Multimedia Networks, WoWMoM, June 2017, pp. 1-9.

[36] C.-L. Wu, L.-C. Fu, F.-L. Lian, WLAN location determination in e-home via support vector classification, in: IEEE International Conference on Networking, Sensing and Control, 2004, vol. 2, 2004, pp. 1026-1031.

[37] V. Honkavirta, T. Perala, S. Ali-Loytty, R. Piche, A comparative survey of WLAN location fingerprinting methods, in: 6th Workshop on Positioning, Navigation and Communication, March 2009, pp. 243-251.

[38] R. Parker, S. Valaee, Vehicular node localization using received-signal-strength indicator, IEEE Trans. Veh. Technol. 56 (6) (Nov 2007) 3371-3380.

[39] A. Khattab, Y.A. Fahmy, A.A. Wahab, High accuracy GPS-free vehicle localization framework via an INS-assisted single RSU, Int. J. Distrib. Sens. Netw. 11 (5) (2015) 795036. [Online]. Available: https://doi.org/10.1155/2015/795036. 
[40] C.Y. Wen, R.D. Morris, W.A. Sethares, Distance estimation using bidirectional communications without synchronous clocking, IEEE Trans. Signal Process. 55 (5) (May 2007) 1927-1939.

[41] N. Zhu, J. Marais, D. Bétaille, M. Berbineau, Gnss position integrity in urban environments: a review of literature, IEEE Trans. Intell. Transp. Syst. 19 (9) (Sep. 2018) 2762-2778.

[42] R.B. Langley, Dilution of precision [Online]. Available: https://pdfs. semanticscholar.org/c04c/d3dc971734ebe2ba1aa113e417e8b28b248c.pdf, May 1999.

[43] Spirent Communications Plc, Fundamental GNSS receiver characterisation [Online]. Available: https://www.spirent.com/resourcelibrarysearchpage.

[44] H. Wymeersch, J. Lien, M.Z. Win, Cooperative localization in wireless networks, Proc. IEEE 97 (2) (Feb 2009) 427-450.

[45] N. Alam, A.G. Dempster, Cooperative positioning for vehicular networks: facts and future, IEEE Trans. Intell. Transp. Syst. 14 (4) (Dec 2013) 1708-1717.

[46] C. Ang, Vehicle positioning using WiFi fingerprinting in urban environment, in: 2018 IEEE 4th World Forum on Internet of Things, WF-IoT, Feb 2018 pp. 652-657.

[47] O. Chia-Ho, A roadside unit-based localization scheme for vehicular ad hoc networks, Int. J. Commun. Syst. 27 (1) (2014) 135-150. [Online]. Available: https:// onlinelibrary.wiley.com/doi/abs/10.1002/dac.2352.

[48] N. Saeed, W. Ahmad, D.M.S. Bhatti, Localization of vehicular ad-hoc networks with RSS based distance estimation, in: 2018 International Conference on Computing, Mathematics and Engineering Technologies, iCoMET, March 2018, pp. 1-6.

[49] P. Oguz-Ekim, K. Ali, Z. Madadi, F. Quitin, W.P. Tay, Proof of concept study using DSRC, IMU and map fusion for vehicle localization in GNSS-denied environments, in: IEEE 19th International Conference on Intelligent Transportation Systems, ITSC, Nov 2016, pp. 841-846.

[50] G. Dissanayake, S. Sukkarieh, E. Nebot, H. Durrant-Whyte, The aiding of a lowcost strapdown inertial measurement unit using vehicle model constraints for land vehicle applications, IEEE Trans. Robot. Autom. 17 (5) (Oct 2001) 731-747.

[51] X. Zhong, R. Rabiee, Y. Yan, W.P. Tay, A particle filter for vehicle tracking with lane level accuracy under GNSS-denied environments, in: 2017 IEEE 20th International Conference on Intelligent Transportation Systems, ITSC, Oct 2017, pp. 1-6.

[52] A. Fascista, G. Ciccarese, A. Coluccia, G. Ricci, A localization algorithm based on V2I communications and AOA estimation, IEEE Signal Process. Lett. 24 (1) (Jan 2017) 126-130.

[53] H. Kloeden, D. Schwarz, E.M. Biebl, R.H. Rasshofer, Vehicle localization using cooperative RF-based landmarks, in: 2011 IEEE Intelligent Vehicles Symposium (IV), June 2011, pp. 387-392.

[54] N. Hernández, A. Hussein, D. Cruzado, I. Parra, J.M. Armingol, Applying low cost WiFi-based localization to in-campus autonomous vehicles, in: 2017 IEEE 20th International Conference on Intelligent Transportation Systems, ITSC, Oct 2017, pp. 1-6.

[55] H. Lu, S. Zhang, Y. Dong, X. Lin, A Wi-Fi/GPS integrated system for urban vehicle positioning, in: 13th International IEEE Conference on Intelligent Transportation Systems, Sept 2010, pp. 1663-1668.

[56] S. Kumar, R.M. Hegde, N. Trigoni, Gaussian process regression for fingerprinting based localization, Ad Hoc Netw. 51 (2016) 1-10 [Online]. Available: http:// www.sciencedirect.com/science/article/pii/S1570870516301834.

[57] S. Yiu, K. Yang, Gaussian process assisted fingerprinting localization, IEEE Int Things J. 3 (5) (Oct 2016) 683-690.

[58] T. Wang, P. Tseng, Y. Chan, D. Lin, A ray-tracing based fingerprinting for indoor positioning, in: 2015 IEEE 12th Intl Conf on Ubiquitous Intelligence and Computing and 2015 IEEE 12th Intl Conf on Autonomic and Trusted Computing and 2015 IEEE 15th Intl Conf on Scalable Computing and Communications and Its Associated Workshops, UIC-ATC-ScalCom, Aug 2015, pp. 1859-1863.

[59] G.M. Hoang, B. Denis, J. Härri, D.T.M. Slock, Breaking the gridlock of spatial correlations in GPS-aided IEEE 802.11p-based cooperative positioning, IEEE Trans. Veh. Technol. 65 (12) (Dec 2016) 9554-9569.

[60] M. Elazab, A. Noureldin, H.S. Hassanein, Integrated cooperative localization for vehicular networks with partial GPS access in urban canyons, Veh. Commun. 9 (2017) 242-253 [Online]. Available: http://www.sciencedirect.com/science/ article/pii/S2214209616300651.
[61] P.D. Groves, Z. Jiang, Height aiding, $\mathrm{C} / \mathrm{N}_{0}$ weighting and consistency checking for GNSS NLOS and multipath mitigation in urban areas, J. Navig. 66 (5) (2013) 653-669.

[62] K. Liu, H.B. Lim, E. Frazzoli, H. Ji, V.C.S. Lee, Improving positioning accuracy using GPS pseudorange measurements for cooperative vehicular localization, IEEE Trans. Veh. Technol. 63 (6) (July 2014) 2544-2556.

[63] G. Zhang, W. Wen, L.T. Hsu, A novel GNSS based V2V cooperative localization to exclude multipath effect using consistency checks, in: 2018 IEEE/ION Position, Location and Navigation Symposium, PLANS, April 2018, pp. 1465-1472.

[64] N.M. Drawil, O. Basir, Intervehicle-communication-assisted localization, IEEE Trans. Intell. Transp. Syst. 11 (3) (Sept 2010) 678-691.

[65] J. Liu, B.g. Cai, J. Wang, Cooperative localization of connected vehicles: integrating GNSS with DSRC using a robust cubature Kalman filter, IEEE Trans. Intell. Transp. Syst. 18 (8) (Aug 2017) 2111-2125.

[66] D.V. Nguyen, F. Nashashibi, T.K. Dao, E. Castelli, Improving poor GPS area localization for intelligent vehicles, in: IEEE International Conference on Multisensor Fusion and Integration for Intelligent Systems (MFI), Nov 2017, pp. 417-421.

[67] N. Dinh-Van, F. Nashashibi, N. Thanh-Huong, E. Castelli, Indoor intelligent vehicle localization using WiFi received signal strength indicator, in: 2017 IEEE MTT-S International Conference on Microwaves for Intelligent Mobility, ICMIM, March 2017, pp. 33-36.

[68] J. Li, X. Cui, Z. Li, J. Liu, Method to improve the positioning accuracy of vehicular nodes using IEEE 802.11p protocol, IEEE Access 6 (2018) 2834-2843.

[69] F.A. Santos, A.T. Akabane, R.S. Yokoyama, A.A.F. Loureiro, L.A. Villas, A roadside unit-based localization scheme to improve positioning for vehicular networks, in: 2016 IEEE 84th Vehicular Technology Conference, VTC-Fall, Sept 2016, pp. 1-5.

[70] L. Xu, S. Zhang, J. Quan, X. Lin, Vehicle positioning using Wi-Fi networks and GPS/DR system, in: Fifth International Conference on Mobile Ad-Hoc and Sensor Networks, Dec 2009, pp. 287-293.

[71] A. Amini, R.M. Vaghefi, J.M. de la Garza, R.M. Buehrer, Improving GPS-based vehicle positioning for intelligent transportation systems, in: IEEE Intelligent Vehicles Symposium Proceedings, June 2014, pp. 1023-1029.

[72] L. Conde, R. Chelim, U. Nunes, Collaborative vehicle self-localization using multi-GNSS receivers and V2V/V2I communications, in: 2015 IEEE 18th International Conference on Intelligent Transportation Systems, Sept 2015, pp. 2525-2532.

[73] A. Fascista, G. Ciccarese, A. Coluccia, G. Ricci, Angle of arrival-based cooperative positioning for smart vehicles, IEEE Trans. Intell. Transp. Syst. (2017) 1-13.

[74] S.B. Cruz, T.E. Abrudan, Z. Xiao, N. Trigoni, J. Barros, Neighbor-aided localization in vehicular networks, IEEE Trans. Intell. Transp. Syst. 18 (10) (Oct 2017) 2693-2702.

[75] C. Ang, Vehicle positioning using WIFI fingerprinting in urban environment, in: IEEE 4th World Forum on Internet of Things, WF-IoT, Feb 2018

[76] S.H. Fang, Y.H.S. Yang, The impact of weather condition on radio-based distance estimation: a case study in GSM networks with mobile measurements, IEEE Trans. Veh. Technol. 65 (8) (Aug 2016) 6444-6453.

[77] S. Taguchi, T. Yoshimura, Robust Bayesian filtering for positioning using GPS INS in multipath environments, in: IEEE/ION Position, Location and Navigation Symposium, PLANS, April 2018, pp. 816-821.

[78] M.L. Cherif, J. Leclère, R.J. Landry, Loosely coupled GPS/INS integration with snap to road for low-cost land vehicle navigation: EKF-STR for low-cost applications, in: IEEE/ION Position, Location and Navigation Symposium, PLANS, April 2018, pp. 275-282.

[79] M. Elbahnasawy, T. Shamseldin, A. Habib, Image-assisted GNSS/INS navigation for UAV-based mobile mapping systems during GNSS outages, in: 2018 IEEE/ION Position, Location and Navigation Symposium, PLANS, April 2018, pp. 417-425.

[80] M. Moussa, A. Moussa, N. El-Sheimy, Multiple ultrasonic aiding system for car navigation in GNSS denied environment, in: IEEE/ION Position, Location and Navigation Symposium, PLANS, April 2018, pp. 133-140.

[81] L. Oliveira, C. Di Franco, T. Abrudan, L. Almeida, Fusing time-of-flight and received signal strength for adaptive radio-frequency ranging, in: 16th International Conference on Advanced Robotics, ICAR 2013, November 2013, pp. 1-6. 